\title{
Mucosa-Associated Lymphoid Tissue
}

National Cancer Institute

\section{Source}

National Cancer Institute. Mucosa-Associated Lymphoid Tissue. NCI Thesaurus. Code C12910.

Lymphoid tissue located beneath the mucosal epithelia of those mucosal surfaces that have contact with the external environment, such as the respiratory, digestive, and urinary systems. MALT consists of a collection of predominantly small lymphocytes, fewer larger, transformed lymphocytes, and plasma cells. It protects the body from pathogens that enter via the mucosa. MALT gives rise to a distinctive type of B-cell lymphoma that usually follows an indolent clinical course. 